\title{
Towards Highest Spectral Efficiency: Optical Sinc-Shaped Nyquist Pulses Generation from Rectangular Frequency Comb
}

\author{
Camille-Sophie Brès ${ }^{1}$, Member, IEEE, Marcelo A. Soto ${ }^{2}$, Mehdi Alem², Mohammad Amin Shoaie', \\ Armand Vedadi ${ }^{1}$, Thomas Schneider ${ }^{3}$, and Luc Thevenaz ${ }^{2}$, Senior Member, IEEE \\ ${ }^{I}$ Ecole Polytechnique Fédérale de Lausanne (EPFL), Photonic Systems Laboratory (PHOSL), \\ Station 11, STI-IEL, CH-1015 Lausanne, Switzerland \\ ${ }^{2}$ Ecole Polytechnique Fédérale de Lausanne (EPFL), Group for Fibre Optics (GFO), SCI-STI-LT, \\ Station 11, CH-1015 Lausanne, Switzerland \\ ${ }^{3}$ Institut für Hochfrequenztechnik, Hochschule für Telekommunikation Leipzig, \\ Gustav-Freytag-Str. 43-45, 04277 Leipzig, Germany \\ Tel: +41(21)6937866,e-mail: camille.bres@epfl.ch
}

\begin{abstract}
In this paper, we review a method to produce optical sinc-shaped Nyquist pulses with unprecedented quality. The method is based on the synthesis of a rectangular shaped and phase-locked frequency comb from a combination of intensity modulators. The result is a highly flexible pulse generator that can easily be integrated in already installed communication systems. All-optical pulse shaping methods for highest spectral efficiencies are attractive since high-bitrate spectrally efficient channels can be combined into multiple Tbit/s superchannels, enabling ultrahigh data transmission rates and a simultaneous ultrahigh spectral efficiency. Ideal sincshaped Nyquist pulses thus unlock the path towards minimal inter-symbol interference and no-guard band wavelength multiplexing. Here we will report on the generation, modulation and multiplexing of the sinc-shaped pulses obtained while using off-the-shelf components.
\end{abstract}

Keywords: all-optical pulse shaping, optical Nyquist communication, frequency combs.

\section{INTRODUCTION}

The continuous increase in the demand for higher data transmission rates has motivated the research of new approaches to improve the spectral efficiency of optical communication networks [1]. Pulse shaping for highest spectral efficiency has attracted the attention of the community such that orthogonal frequency division multiplexing (OFDM) [2], and Nyquist multiplexing amongst other schemes have emerged [3]. All-optical approaches are attractive to combine high-bitrate spectrally efficient channels into multiple Tbit/s superchannels, enabling ultrahigh data transmission rates and a simultaneous ultrahigh spectral efficiency. With OFDM, a data rate of $26 \mathrm{Tbit} / \mathrm{s}$ and a net spectral efficiency of $5 \mathrm{bit} / \mathrm{s} / \mathrm{Hz}$ have been demonstrated [4]. Recently, a $32.5 \mathrm{Tbit} / \mathrm{s}$ Nyquist WDM transmission with symbols carried by Nyquist pulses [5], with a net spectral efficiency of $6.4 \mathrm{bit} / \mathrm{s} / \mathrm{Hz}$ has been shown [6].

Nyquist pulses can be orthogonally multiplexed in a wavelength division multiplexing scheme (Nyquist WDM), or in a time division multiplexing scheme (orthogonal TDM) [7]. While orthogonal TDM can communicate most reliably by use of any Nyquist pulses with minimum inter-symbol interference (ISI), a spectrally-efficient Nyquist-WDM transmission requires no guard band between adjacent frequency channels. These two independent properties can only be achieved simultaneously by sinc-shaped Nyquist pulses and such exciting prospects have led to intense research activities. Several approaches for the generation of Nyquist pulses have been suggested. Digital domain shaping was performed with an arbitrary waveform generator programmed offline to create Nyquist filtering of the baseband signal [3]. However, this method is limited by the speed of the electronics whereas the quality of the Nyquist pulses highly depends on the generator sampling rate. All optical generation, which enables optical sequences with much shorter time duration, has been investigated. Liquid crystal spatial modulators have been used to shape Gaussian pulses from a mode-locked laser into raised-cosine Nyquist pulses [8]. It is also possible to generate Nyquist pulses using a fibre optical parametric amplification (FOPA), pumped by parabolic pulses, and a phase modulator to compensate the pump-induced chirp [9]. However none of the pulse shaping methods reported so far have led to close to ideal sinc pulses and most of them have remained rather complex. So, it has not yet been established whether such pulses could be incorporated in optical networks in cost-effective ways.

Here a method to produce sinc-shaped Nyquist pulses reaching perfection is presented [10]. The pulse generation is based on the direct synthesis of a flat, rectangular-shaped and phase-locked frequency comb using a simple combination of intensity modulators. The method is highly flexible and can be easily integrated in communication systems, potentially offering a substantial increase in data transmission rates [11]. 


\section{PROPOSED METHOD}

The data transmitted along a communication link can be distorted when adjacent symbols interfere, resulting in inter-symbol interference (ISI) [12]. This unwanted phenomenon induces errors in signal detection and makes the communication less reliable. Shaping of the data pulses according to the Nyquist criterion is a proven technique to minimized ISI. According to this criterion, the data pulse $r(t)$ is equal to 0 for all sampling time $n T_{S}$, with $n$ an integer and $T_{S}$ the sampling period, except at time origin where $r(0)=1$. Raised-cosine pulses satisfy the Nyquist ISI criterion, and are among the most used pulses in telecommunications. The temporal shape is given by equation 1 .

$$
r(t)=\operatorname{sinc}\left(\frac{\pi t}{T_{S}}\right) \frac{\cos \left(\beta \pi t / T_{S}\right)}{1-\left(2 \beta t / T_{S}\right)^{2}}
$$

where $\beta$, the roll-off factor such that $0 \leq \beta \leq 1$, is a measure of the pulse excess bandwidth with respect to the ideal sinc pulse obtained when $\beta=0$ which has the minimal bandwidth usage of $1 / T_{S}$.
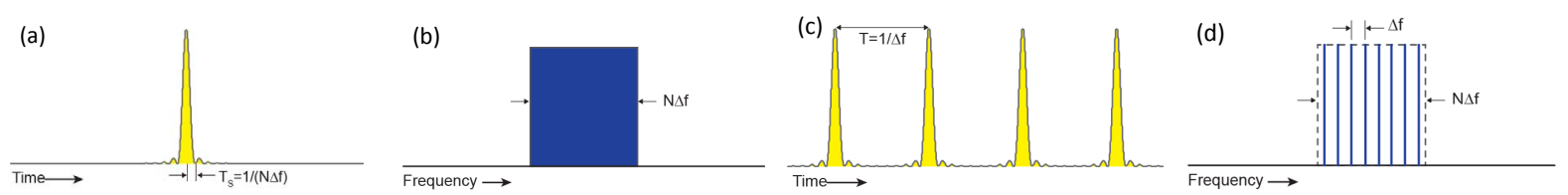

Figure 1. Time and frequency representation of a single-sinc pulse ( $a, b$, respectively) and a sinc-pulse sequence (c, $d$, respectively).

In the Fourier representation, a sinc pulse corresponds to a rectangular spectral function, and therefore, a periodic train of sinc pulses corresponds to a rectangular-shaped and phase-locked frequency comb as shown in Fig. 1 [10]. A train of sinc-shaped Nyquist pulses can thus be directly obtained from the synthesis of a rectangular-shaped optical frequency comb. The simplicity and flexibility of this approach leads to the generation of easily reconfigurable sinc-shaped pulses without loss of quality.

\section{EXPERIMENTAL SETUP}

The experimental setup is shown in Fig. 2. It simply consists in two cascaded Mach-Zehnder modulators driven by synchronized microwave generators. An external cavity laser (ECL) operating at $1550 \mathrm{~nm}$ is sent to the first modulator which is driven with a frequency $f_{1}$. The modulated light then goes to the second modulator driven with a frequency $f_{2}=f_{1} / 3$. The relative phase between both RF signals is carefully adjusted to reach the sought after almost ideal sinc-shaped Nyquist pulses. A semiconductor amplifier (SOA) and tunable band pass filter (TOF) are positioned between the two modulators as to increase the signal to noise ratio (SNR) of the generated pulse train. At the output of the Nyquist generator, the signal is amplified with an erbium doped fiber amplifier (EDFA) and sent to the monitoring section, which consist of an optical sampling oscilloscope with $500 \mathrm{GHz}$ bandwidth and an optical spectrum analyser. Additionally, a third modulator driven by a pseudo random bit sequence (PRBS) can be positioned after the EDFA for data encryption onto the sinc-shaped pulse train. It is worth noting that the bandpass filters used in the experimental setup are solely used for noise filtering purposes and not for pulse shaping.

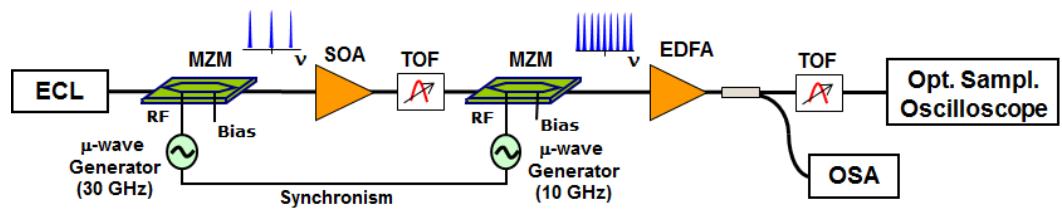

Figure 2. Experimental setup for high quality sinc-shaped modulated Nyquist pulse generation.

\section{EXPERIMENTAL RESULTS AND DISCUSSION}

As a proof of concept, rectangular frequency combs with 9 lines centered at $1550 \mathrm{~nm}$ were generated using the setup presented in Fig. 2. Two synchronized microwave generators are used to drive the two cascaded MachZehnder modulators. For this demonstration the frequency of the first generator was set to $30 \mathrm{GHz}$, while the second modulator was driven at $10 \mathrm{GHz}$ resulting in a frequency combs with spectral components spaced at $\Delta f=10 \mathrm{GHz}$ as shown in Fig. 3a. Spectral flatness within $1 \mathrm{~dB}$ was obtained together with a high rejection (at least $27 \mathrm{~dB}$ ) of out of band components. The overall comb spans $90 \mathrm{GHz}$. In the time domain, a sequence of sinc-shaped Nyquist pulses is observed with a repetition period of $1 / \Delta f=100 \mathrm{ps}$ and a peak-to-zero crossing of $11.1 \mathrm{ps}$, equivalent to a full width half maximum (FWHM) of $9.8 \mathrm{ps}$ (Fig. 3c). One notable quality of this simple 
approach is its high tunability. The RF modulating frequencies were changed over 4 orders of magnitude (from $f_{1}=30 \mathrm{MHz}$ to $f_{1}=30 \mathrm{GHz}$ ), resulting in an easily modified comb line separation and comb bandwidth. The obtained pulses showed a fully similar perfect sinc shape over the entire 4 decade of frequency tuning. An illustration of the pulse train measured in the case of $f_{1}=30 \mathrm{MHz}$ and $f_{2}=10 \mathrm{MHz}$ is shown in Fig. $3 \mathrm{~d}$. The experimentally obtained waveform is almost undistinguishable from the $\mathrm{GHz}$ case, except for the time axis scaling. Such high tunability is not feasible with other techniques, for which the sharpness of the filter used to shape the pulses typically varies with the bandwidth. A colour grade rendering of the 9.8 ps FMWH pulse from the $90 \mathrm{GHz}$ wide comb is shown in Fig. 3b, enabling the monitoring of jitter and signal to noise ratio. For all analysed cases (from $\mathrm{MHz}$ to $\mathrm{GHz}$ rates), jitter and SNR resulted to be below $82 \mathrm{fs}$ and more than $40 \mathrm{~dB}$, respectively. Finally the root mean square (RMS) error between the measured shapes and theoretical shape given by equation 1 was evaluated as a function of the roll-off factor $\beta$. It was found that the RMS error is minimized within $1 \%$ for a roll off factor of 0 . This result indicates that the experimentally obtained pulse shapes are very well fitted with the theoretical ideal sinc shape.
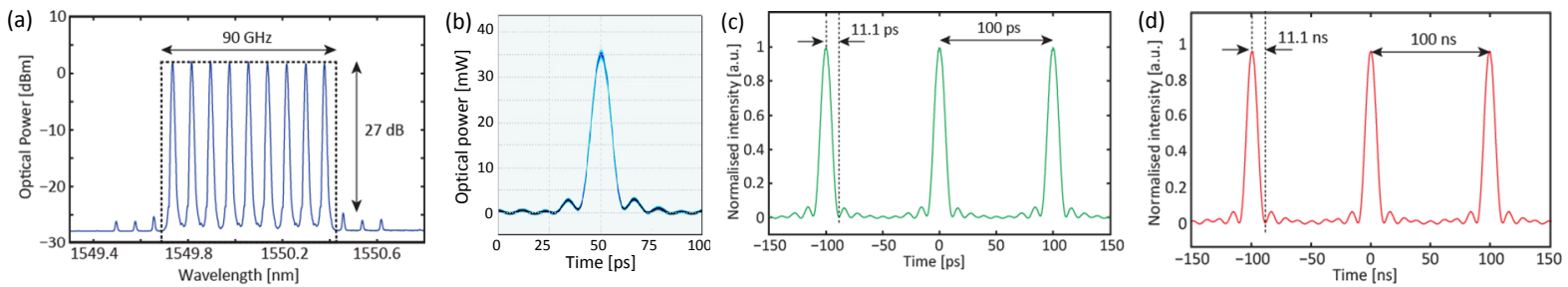

Figure 3: (a) Spectrum of the generated flat comb composed of 9 spectral components spaced by 10 GHz, spanning $90 \mathrm{GHz}$; (b) Colour grade figure of the measured pulse corresponding to the spectrum given in a); (c) Time domain waveform corresponding to the spectrum given in a); (d) Time domain waveform corresponding to a comb generated with 9 lines separated by $10 \mathrm{MHz}$, spanning $90 \mathrm{MHz}$.

Data can be directly encoded onto the sequence of sinc-shaped Nyquist pulse and a modulator driven by the desired data pattern can be inserted before or after the Nyquist generation setup shown in Fig. 2. As a demonstration, data was encoded onto the 9.8 ps FWHM sinc-shaped pulses using a pseudo-random binary sequence (PRBS) with a $2^{31}-1$ length. Figure $4 \mathrm{a}$ shows the measured spectrum resulting from modulating the obtained sinc pulses with OOK modulation format. The experimental results were compared to some theoretical predictions, leading to an almost perfect agreement. The eye of the modulated sinc-shaped Nyquist sequence is shown in Fig. 4b. The quality of the sinc-shape pulse is maintained and a clear eye opening is observed. As is expected, the spectrum of the original frequency comb broadens as a result of the modulation. However, about $99 \%$ of the power still remains within the main spectral lobe, such that the resulting broadening is evaluated to be only $11 \%$. This corresponds to a roll-off factor equal to $\beta=1 / N=0.11, N$ being the number of spectral lines within the comb (i.e. 9 in this case). It should be noted that this broadening is significantly lower than the one obtained by other optical pulse shaping methods even for unmodulated pulses. Since ideal sinc pulses maintain the information within the ideal Nyquist bandwidth, the spectral broadening reported here can be still considerably reduced by increasing the number of spectral lines within the comb. With the presented technique, increasing the number of spectral lines is easily accomplished by modifying the input RF signals to the modulators without any modifications to the hardware part of the setup. For instance, using two RF signals to drive one of the EOMs, a frequency comb having $N=15$ lines can be generated, resulting in an improved roll-off factor down to 0.067 .
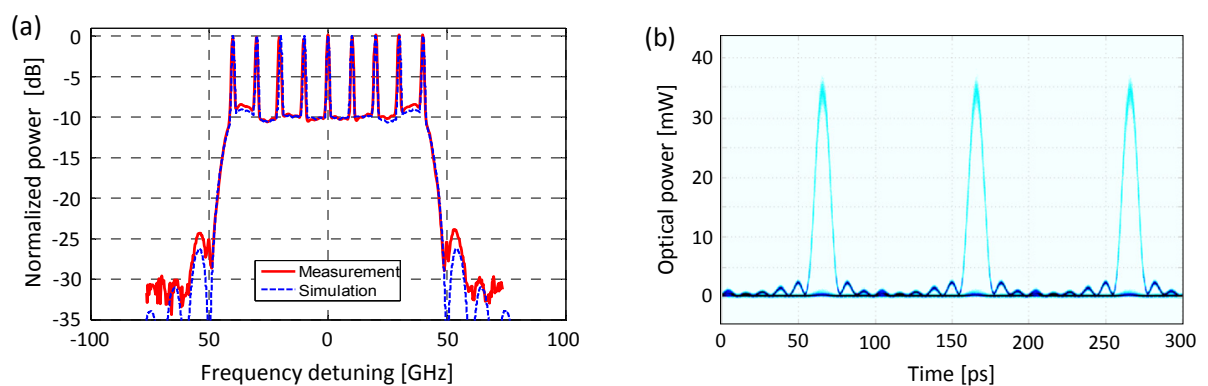

Figure 4: (a) Measured spectrum of the modulated pulses with OOK format (full red line) and simulation (dashed blue line); (b) Colour grade figure of the modulated sinc-shaped Nyquist pulse train.

The generation of close to ideal sinc-shaped pulses has a great potential in increasing the spectral efficiency since the obtained channel possess a rectangular spectrum. Such property is indeed a crucial step towards spectrally-efficient Nyquist-WDM transmission with no guard band between adjacent frequency channels. 
We first tested the operating range of our Nyquist shaper by tuning the input signal wavelength over $5 \mathrm{~nm}$ without adjusting the settings to the modulators. As seen in Fig. 5a, similar pulse trains were obtained demonstrating that only two modulators can be used to simultaneously generate sinc-pulse sequences at different wavelengths. We then concurrently generated two Nyquist channels by directly sending two independent seeds through the Nyquist pulse generation architecture shown in Fig. 2. Both carriers were simultaneously shaped by the two intensity modulators to generate 9 lines per channel. When the two carriers were positioned exactly $90 \mathrm{GHz}$ apart, a two channel Nyquist-WDM composite signal without guard band was obtained as shown in Fig. $5 b$.

(a)

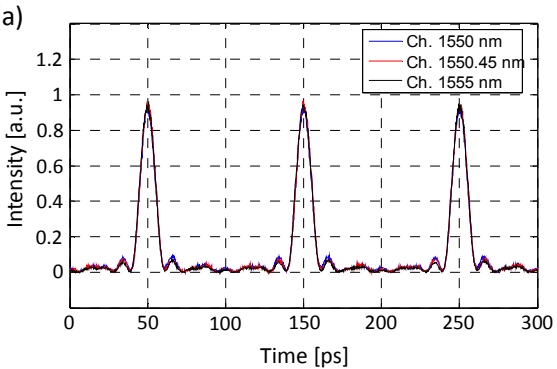

(b)

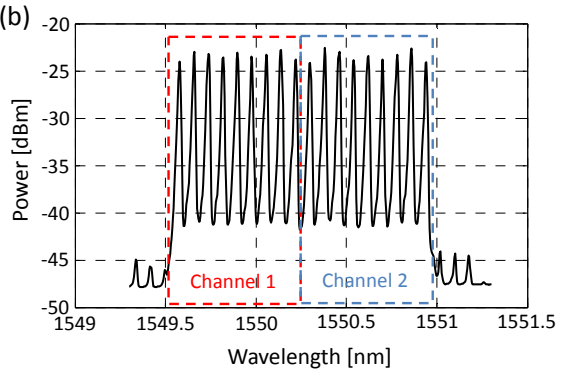

Figure 5: (a) Time domain waveform corresponding of three Nyquist channels generated from a single cascade of modulator; (b) Spectrum of two multiplexed Nyquist channels with no guard band.

\section{CONCLUSIONS}

In conclusion, we have reviewed a method to produce optical sinc-shape Nyquist pulses with unprecedented quality. The method is broadly tunable and highly reconfigurable while utilizing only off-the-shelf components. Close to zero roll-off factors, low jitter and high SNR have been verified, demonstrating the capabilities of the proposed method. The effect of data modulation on the pulse spectrum has been experimentally verified for OOK modulation format, leading to a very low spectral broadening in comparison to other reported optical pulse shaping methods. The close to ideal shape of the pulses represents a step closer towards low cost easily implementable optical transmission with highest spectral efficiency.

\section{REFERENCES}

[1] C. Schubert et al.: New trends and challenges in optical digital transmission systems, in Proc. European Conference and Exhibition on Optical Communication (ECOC 2012), paper We.1.C.1.

[2] Reference om OFDM

[3] S. Schmogrow et al:: Real-time Nyquist pulse generation beyond $100 \mathrm{Gbit} / \mathrm{s}$ and its relation to OFDM, Opt. Express 20, 317-337 (2012).

[4] D. Hillerkuss et al:: 26 Tbit/s line-rate super-channel transmission utilizing all-optical fast Fourier transform processing, Nat. Photonics 5, 364-371 (2011).

[5] H. Nyquist: Certain topics in telegraph transmission theory. Trans. Am. Inst. Electr.Eng. 47, 617-644 (1928).

[6] D. Hillerkuss et al:: Single-laser 32.5 Tbit/s Nyquist WDM transmission, J. Opt. Comm. Netw.4, 715-723 (2012).

[7] M. Nakazawa et al.: Ultrahigh-speed "orthogonal" TDM transmission with an optical Nyquist pulse train, Opt. Express 20, (2012).

[8] H.N. Tan et al.: No guard-band wavelength translation of Nyquist OTDM-WDM signal for spectral defragmentation in an elastic add-drop node, Opt. Letters, 39, (2013)

[9] A. Vedadi, M.A. Shoaie, and C.-S. Brès: Near-Nyquist optical pulse generation with fiber optical parametric amplification, Opt. Express 20, (2012).

[10] M.A. Soto et al.: Optical sinc-shaped Nyquist pulses of exceptional quality, Nature Communications, 4 Dec. 2013.

[11] M.A. Soto et al.: Optical sinc-shaped Nyquist pulses with very low roll off generated from a rectangular frequency comb, in ACP: 2013, OSA Technical Digest (online) (Optical Society of America, 2013), paper PD AF2E.

[12] J. G. Proakis and M. Salehi: Digital Communications, Fifth Edition, McGraw-Hill, 2008. 